\title{
LA ESCRITURA AUTÓGRAFA DE SCHILLER Y SU \\ PENSAMIENTO ENTRE 1792 Y 1794 (UNA APROXIMACIÓN).
}

\author{
SCHILLER'S AUTOGRAPH WRITING AND HIS THOUGHT \\ BETWEEN 1792 AND 1794 (AN APPROXIMATION).
}

Miguel Salmerón Infante

Universidad Autónoma de Madrid

\section{ABSTRACT}

This article attempts to study the thought of Friedrich Schiller between 1792 and 1794 through his autograph writing. For this we have used the author's handwritten texts digitized by the Goethe- und Schiller-Archiv in Weimar. Examining the deletions, corrections and additions of the writing, we try to approximate the author's thought during the mentioned period. This is a watershed moment in Schiller's evolution. Kantian concepts are already well established in it, as demonstrated by the determination of his writing on practical and aesthetic philosophy. However, writing about literary genres, more subject to correction, reveals a process of change. We interpret these differences in writing as Schiller's attempt to make a compendium of the influence that Kant's philosophy had on him and the beginning of a fruitful intellectual relationship with Goethe.

Keywords: Schiller, autograph writing, digitized file, Kant, Goethe. 


\section{RESUMEN}

Este artículo intenta estudiar el pensamiento de Friedrich Schiller entre 1792 y 1794 a través de su escritura autógrafa. Para ello nos hemos servido de textos manuscritos del autor digitalizados por el Goethe- und Schiller-Archiv de Weimar. Examinando las tachaduras, las correcciones y los añadidos de la escritura intentamos aproximarnos al pensamiento del autor durante el citado periodo. Este es un momento decisivo en la evolución de Schiller. En él ya están bien asentados los conceptos kantianos, tal y como demuestra lo resuelto de su escritura sobre filosofía práctica y estética. Sin embargo, la escritura sobre los géneros literarios, más sometida a corrección revela un proceso de cambio. Estas diferencias en la escritura las interpretamos como el intento de Schiller de hacer un compendio de la influencia que ejerció en él la filosofía de Kant y el comienzo de una fructífera relación intelectual con Goethe.

Palabras clave: Schiller, escritura autógrafa, archivo digitalizado, Kant, Goethe.

Fecha de recepción: 14 de junio de 2021.

Fecha de aceptación: 14 de julio de 2021.

Cómo citar: Salmerón Infante, Miguel (2021): «La escritura autógrafa de Schiller y su pensamiento entre 1792 y 1794 (una aproximación)», en Actio Nova: Revista de Teoría de la Literatura y Literatura Comparada, 5: 61-75.

DOI: https://doi.org/10.15366/actionova2021.5.003 
El Archivo Goethe y Schiller de Weimar, lleva desde 2012 realizando la digitalización de los textos originales ${ }^{1}$ (o en su caso de copias manuscritas por parte de otras personas cercanas) no solo de los escritos de estos autores sino también de algunos anteriores (Wieland) y otros posteriores (Liszt y Nietzsche) ${ }^{2}$.

Intentar acceder a la evolución del pensamiento de un autor a través de su escritura autógrafa, es una empresa que lleva consigo una enorme dualidad. Por un lado, no hay un documento más cercano a ese pensamiento que la escritura de puño y letra. Por otro, no dejamos de movernos en el ámbito de la especulación cuando intentamos pasar de la escritura al pensar, incluso al sentir que impulsa aquella.

Bien conocido es que, en Alemania ${ }^{3}$, desde principios de la Edad Moderna a mediados del siglo XX, la Kurrentschrift era el proceder habitual para las cartas y los documentos oficiales (Beck 1991:454). El empleo de una letra cursiva, con una simplificación de los trazos, se entendía como un medio mucho más rápido de pasar de lo pensado a lo escrito. En el fondo aquí encontramos una convención adicional para la escritura. Sin embargo, en los trabajos interpretativos, como este, las convenciones no han de ser vistas de un modo estrictamente negativo. Es cierto que la convención mitiga o incluso sofoca la espontaneidad, pero puede que la imposición que establece no siempre sea asumible y provoque alguna reveladora reacción aversiva en el sujeto. Es posible que en las líneas anteriores resuene la concepción que Freud tenía de la libre asociación o de las diversas teorías sobre la escritura automática, pero en todo caso creemos que la pista que ofrecen esas tradiciones es acertada.

El caso de Schiller puede ser paradigmático de esa refractariedad a la norma, si queremos llamarlo así, de ese malestar en la cultura. Muchas biografías lo describen como sanguíneo y nervioso. Leer sus cartas, sin atender nada más que a su contenido, transmite la impresión de haber sido redactadas por un hombre para el que el tiempo pasaba demasiado

\footnotetext{
${ }^{1}$ Este artículo incluye la reproducción de dos fragmentos de escritura autógrafa de Schiller procedentes respectivamente de dos archivos del Goethe- und Schiller- Archiv de Weimar, a saber, del GSA 83/86 y del GSA 83/85.

2 Todos ellos tienen una presencia muy relevante en la ciudad de Weimar. Wieland fue preceptor de los hijos de la Duquesa Anna-Amalia. Goethe fue consejero áulico del Archiduque Carlos-Augusto. Schiller pasó sus últimos años en Weimar donde escribió sus mejores obras dramáticas. Liszt fue maestro de capilla de la corte entre 1843 y 1861 y director musical del Teatro Nacional que tiene su sede en la ciudad. Salvo Liszt, los otros cuatro murieron en Weimar.

${ }^{3} \mathrm{Al}$ emplear aquí el término «Alemania» nos referimos a la nación cultural, no a la político-estatal, que procede de 1871.
} 
deprisa, alguien que no quería que nada de lo emprendido quedara sin realización. Una incierta situación profesional y una salud muy frágil parecen corroborar que se trataba de un individuo que operaba contrarreloj y sentía tener una espada de Damocles pendiendo sobre su cabeza (Hinderer, 2009: 63). Obtenemos una impresión totalmente diferente si comparamos un manuscrito de Schiller con uno de Goethe. Al consejero áulico se le percibe mucho más adaptado a la convención ${ }^{4}$, a la cursiva y los trazos de la escritura Kurrent. Sin embargo, en Schiller, hay momentos en los que la redonda latina emerge, y esos momentos parecen indicarnos que ahí, ante el aflorar del pensamiento buscado, la convención no puede sostenerse más.

Vamos a fijarnos en varios textos digitalizados de Schiller. Concretamente, cinco que fueron ordenados en la Schiller Nationalausgabe, bajo el rótulo «Aus dem Nachlass» (es decir propios «del legado póstumo»). Materialmente los escritos parecen emparentados pues todos tienen una marca de agua idéntica (Petzold, 2005: 876). Las ediciones canónicas sitúan la redacción de todos ellos entre 1792 y 1794 (Wiese, 1963: 389), años decisivos para el autor, pues ya había superado una grave crisis de salud, ya había leído a Kant, de entonces datan sus lecciones sobre Estética ${ }^{5}$, ya había redactado las que pasarían a llamarse Augustenburger Briefe $^{6}$ y había refundido y ampliado las ideas desarrolladas en Sobre la educación estética del ser bumano en una serie de cartas $^{7}$ y a partir de 1794 comienza su fructífera relación con Goethe.

De los cinco textos que vamos a tratar, diremos que los comentaristas más reputados, señalan que los dos primeros son preparatorios de Sobre poesía ingenua y sentimental, mientras que los tres últimos delinean las cartas Sobre la educación estética (Janz, 1992: 1550).

\footnotetext{
${ }^{4}$ No hay que olvidar que Goethe contó con mucha más frecuencia y continuidad con secretarios (Riemer, Eckermann) que Schiller. Lo más llamativo, y ciñéndonos a sus relaciones con mujeres, es la enorme influencia de las diferencias sociales en su práctica de escritura. Mientras que a la patricia y anhelada Charlotte von Stein siempre le escribió de puño y letra, para su esposa Christiane Vulpius dictaba sus cartas y le ponía unas notas al final. Que la intimidad existiera en el primer caso y fuera ignorada en el otro es desde nuestros parámetros actuales sorprendente

${ }^{5}$ Desarrolladas en Jena el semestre de invierno de 1792-3. De ellas sacó apuntes Christian Friedrich Michaelis que las publicó como apéndice a Geist in Scbillers Werken, gesammelt von Ch.F.M., Leipzig, 1806.

${ }^{6}$ En 1793, Schiller recibió una ayuda económica de tres años de duración y a razón de 1000 táleros anuales de parte de Friedrich Christian von Schleswig-Holstein-Sondenburg-Augustenburg. Como agradecimiento el autor hace un compendio de su pensamiento estético en forma de cartas que envía al Duque. En 1794 un incendio en el Palacio Real de Copenhague destruye las cartas. Por ello, el Duque le solicitó al autor una copia. La primera edición impresa data de 1876 en dos números de Deutsche Rundschau. El 7 (pp.67-81; pp.273-284; pp.400-413) y el 8 (pp.253-268).

${ }^{7}$ La primera edición tuvo lugar en la revista Die Horen en tres números publicados en 1795, concretamente el 1 (Cartas 1-9, pp.7-48), el 2 (Cartas 10-16, pp.51-94) y el 6 (Cartas 17-27, pp.15-124). Frente a las Augustenburger Briefe que tenían al Duque por destinatario, estas van dirigidas a alguien anónimo y genérico.

${ }^{8}$ Barthold Petzold nos recuerda que el segundo párrafo de la digitalización 70153_2 de «Tragödie und Komödie» desde Welche von beide... hasta ....zwingt se reproduce exactamente en «Über naive und sentimentalische Dichtung» (Petzold, 2005: 878).
} 
«Tragödie und Kogmödie»" ${ }^{9}$ se trata de un ejemplo muy significativo, porque tanto el borrador como la puesta en limpio para su ulterior impresión (que no tendría lugar más que póstumamente y en el tomo 21 de la Nationalausgabe en sus páginas 91 a 93) son del propio escritor.

Para empezar, recordemos que estamos en el momento álgido de influencia de Kant en Schiller. Aunque a raíz de su enfermedad pulmonar y su necesidad de reposo, a partir de 1791 Schiller leyó las tres críticas de Kant, sin duda alguna la que más le influyó fue la Crítica de la facultad de juzgar, y, muy especialmente, la «Crítica del Juicio estético». Conforme a ello, Schiller subraya tanto en el borrador como en la copia, los conceptos fundamentales, claramente influido por el inspirador de su pensamiento estético en este momento de su obra.

El texto comienza afirmando que ambas, comedia y tragedia tienen como objetivo liberar al ánimo o al alma (Gemüt), pero la comedia lo consigue mediante la indiferencia moral y la tragedia mediante la autonomía. Y precisamente esos dos términos moralische Indifferenz y Autonomie son subrayados. Y lo son tanto en el borrador como en la copia en limpio (y así será con todas las palabras que mencionemos hasta que avisemos de lo contrario).

En la comedia, prosigue Schiller, lo moral ha de ser conducido a lo físico, pues los asuntos morales no permiten ninguna indiferencia ${ }^{10}$. Así si la comedia introduce algo que interese a nuestros sentimientos morales, debe neutralizarlo, es decir llevarlo al ámbito de lo físico. Y es precisamente neutralisieren el término subrayado en este párrafo.

Casi sin solución de continuidad, Schiller subraya Undank (desagradecimiento o ingratitud). Cuestión, por cierto, candente en aquellos estertores de la Edad Moderna, en la que intelectuales como Goethe o Schiller todavía dependían de sus protectores (como Carlos Augusto de Weimar-Sachsen) ${ }^{11}$. De ahí que todavía fuera un dilema ser agradecido o reivindicar la ansiada independencia mediante un desdén todavía reprimido. En este sentido es enormemente significativo que Undank sea el comienzo del tercer párrafo del texto autógrafo.

\footnotetext{
${ }^{9}$ https://ores.klassik-stiftung.de/ords/f?p=401:2:::::P2_ID:70153 (último acceso: 01/05/2021)

${ }^{10}$ Y la comedia nos quiere llevar a una situación de distanciada y serena indiferencia.

${ }^{11}$ No olvidemos que Schiller y Goethe viven al final de la Edad Moderna y al principio de la contemporánea. Tanto en uno como en otro hay una enorme ambigüedad en torno a esta cuestión de las diferencias estamentales. Si bien abogan por una nivelación democrática de la sociedad, no dejan de asumir los honores que la nobleza de la época les otorgó. Es muy significativo que ambos lograran que se les incluyera en su apellido el nobiliario von.
} 
En todo caso, y retornando a la argumentación schilleriana, el desagradecimiento o ingratitud de las hijas de Lear es trágico y debe herirnos, porque la tragedia nos exige sufrir. $\mathrm{Y}$ ese sufrimiento no puede y no debe sernos ahorrado o evitado. Aquí es muy significativo que en el mismo texto Schiller añade al no «puede» (kann) el ni «debe» (und soll) a modo de superíndice volado mediante una llave. Acto seguido y conforme a ello leiden («sufrir») es la siguiente palabra subrayada [Imagen 1]. Ese sufrimiento ha de ser entendido al hilo de la lectura que Schiller hace de Kant, de lo sublime dinámico (renombrado por Schiller como sublime práctico). Si nos agrada la tragedia a pesar de que en ella vemos sufrimiento es porque el sufrimiento nos conduce a la libertad. Es decir, el sentimiento de libertad del héroe, compartido por nosotros, está por encima de cualquier sufrimiento físico o anímico infligido.

Muy diferente es la introducción de la ingratitud en la comedia, la cual si quiere atenerse a su código ha de mostrarnos ridículo al que pretende agradecimiento sin obtenerlo. En este sentido es muy interesante la corrección en el borrador, en lugar del für, para expresar que la ingratitud debe ser tomada en la tragedia «por algo natural» (eine natürliche Sache), se pone als («como algo natural»), fortaleciendo la neutralización de lo moral y acentuando lo físico que debe ser propio de los asuntos de la comedia. ¿Se puede decir que la apreciable cantidad de correcciones está relacionada con el hecho de que este escrito era un esbozo de «Sobre poesía ingenua y sentimental»? No olvidemos que el texto final iba a ser expuesto al escrutinio de intelectuales de renombre del pertenecientes al consejo editorial de Die Horen ${ }^{12}$. ¿Puede explicarse por eso las numerosas modificaciones? De nuevo solo se puede decir que esta es una hipótesis probable, pero no podemos convertirla en una afirmación concluyente.

\footnotetext{
12 Por otra parte, «Sobre poesía ingenua y sentimental» es un texto decisivo en la obra de Schiller, pues, por un lado, se mueve en los parámetros de la estética idealista kantiana (el sentimiento y el juicio estético no procede de las cualidades del objeto, sino de la dinámica inducida por la propia experiencia estética), y, por otro es un claro intento de Schiller de distinguir su modo de hacer poesía del propio de Goethe. De algún modo, podemos decir que es el último escrito kantiano de Schiller y el primero goethiano. Las fechas de publicación en Die Horen entre 1795 y 1796 son muy significativas. Recordemos que la «fiebre Goethe» se había iniciado en Schiller en 1794.
} 


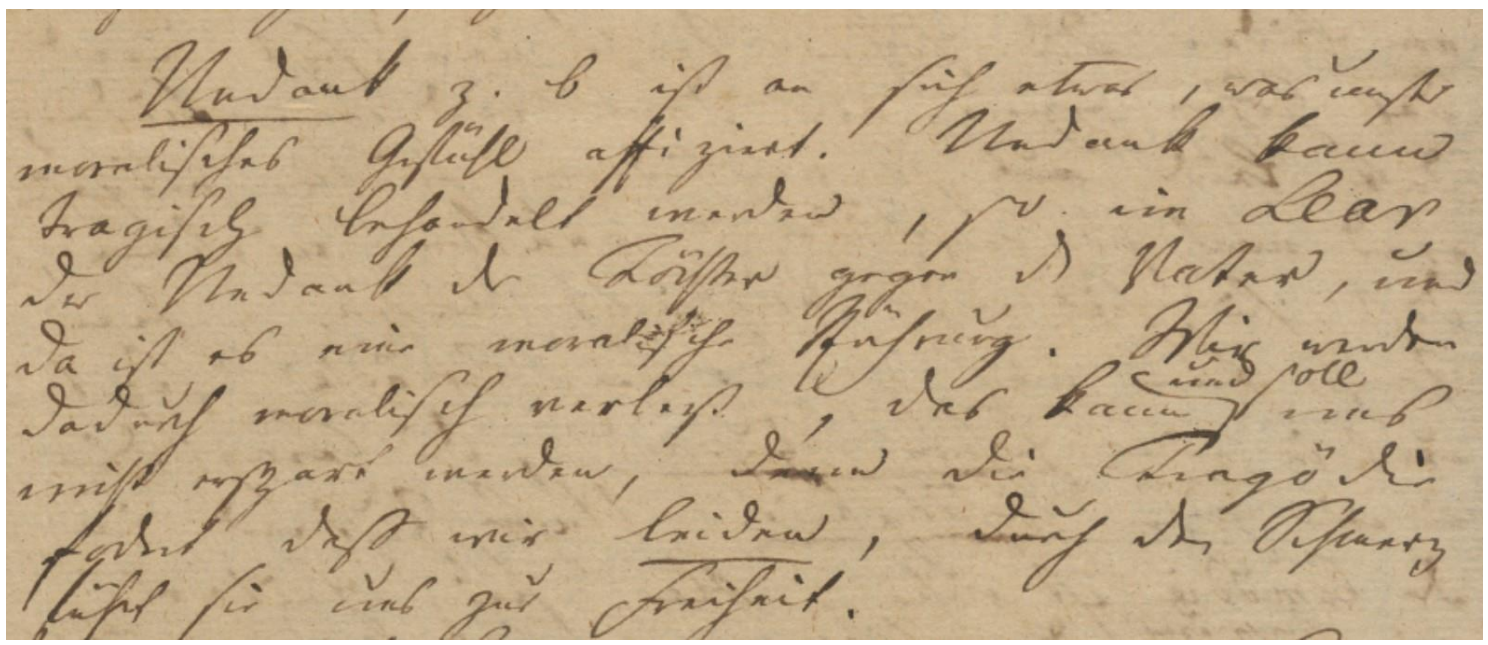

Imagen 1/ Foto: Klassik Stiftung Weimar.

Si El rey Lear es el ejemplo elegido de tragedia, en el muy simétrico texto de Schiller la comedia está representada por Tartufo. El autor señala que a Molière se le ha reprochado haber convertido a ese personaje en protagonista de la obra. Ahora bien, mientras que en el borrador se caracteriza a Tartufo como Gaukler («estafadon»), en la versión definitiva su descripción es de Heuchler («hipócrita»). En todo caso, su argumentación en favor de un género, la comedia en el que la moralidad no desempeña un papel nuclear queda acto seguido reflejada. La maestría de Molière para la comedia queda de manifiesto en que no presenta como repelente o el abominable al hipócrita (subraya er para referirse a este), sino a aquellos a los que engaña (y subraya die para hacer alusión a estos).

Lleva Schiller el hilo del discurso a un punto más genérico cuando se cuestiona. como otros muchos han hecho, si es mejor la comedia o la tragedia. Esta es una pregunta que casi considera retórica, pues entiende que una y otra parten de tan distintos puntos de vista y tienden a efectos tan diversos que no pueden compararse (Schiller, 1963: 92). De nuevo, hay una corrección reveladora. Al principio de la cláusula, el tachado auf es sustituido por aus. Está claro, porque así queda de manifiesto en el borrador y en la versión para imprimir que Schiller dijo que tragedia y comedia "parten de puntos de vista tan distintos» (aus so verschiedenen Punkten ausgehen). Lo que es menos claro y sin duda más interpretativo es cuál sería la cláusula que le vino primero a la cabeza a Schiller e iría de la mano de auf. ¿Auf so verschiedenen Basis (Basen, Boden...) stehen? No puede llegar a ser terminante lo que apuntemos aquí.

A pesar de la imposibilidad de establecer la superioridad de un género sobre otro, Schiller indica y para enfatizarlo subraya que la comedia nos lleva a un «estado más elevado» 
(setżt uns in einen böheren Zustand) y la tragedia a una actividad más elevada (böhere Tätigkeigt). Es decir, la comedia nos conduciría a una indiferencia o un distanciamiento olímpico respecto a lo que nos muestra. Nuestro estado en la comedia es «tranquilo, claro, libre, sereno, no actuamos, ni padecemos, miramos en torno a nosotros y todo nos es externo» (Schiller, 1963: 92).

El lugar, propio de dioses, al que nos lleva como lectores la comedia, no es el modus vivendi, operandi o essendi del ser humano ${ }^{13}$. Es más real estar sometidos al destino ${ }^{14} \mathrm{o}$ a las leyes ${ }^{15}$. Por eso la tragedia contiene un elemento más real, o si se antoja realista, no nos hace dioses, nos hace héroes. Y he aquí el momento en el que la escritura de Schiller se hace más emocional, pues tanto en el borrador como en la copia en limpio para impresión, en lugar de la Kurrentschrift, utiliza la redonda latina para escribir Heroen. Finalmente, el texto se refiere a las figuras heroicas, esas que pueden ser trágicas, los humanos deiformes y los dioses que sufren, los titanes. Aunque más bien parece estar aquí Schiller refiriéndose a los titanes. No es casual que, sin solución de continuidad, el mencionado sea Prometeo al que Schiller considera el símbolo mismo de la tragedia. Y aquí hay de nuevo un punto irresoluble en la escritura. Mientras que, en el borrador, sin titubeos, se escribe Prometheus, en la copia en limpio Schiller comienza escribiendo una palabra que tacha y de la que lo único distinguible es una «m» mayúscula. ¿Mein Prometheus? ¿'Tenía Schiller la intención de honrar al símbolo de la tragedia escribiendo un drama o un poema sobre él? De nuevo, nada se puede afirmar de un modo concluyente.

Sin duda alguna, tributario de las reflexiones sobre tragedia y comedia es el escrito «Nathan der Weise» ${ }^{16}$. Si en el texto anterior se consideran modélicos como tragedia El rey Lear y como comedia Tartufo, aquí Natán el sabio de Lessing es sometido a una acerba crítica. Señala Schiller, que por un momento el Saladino del drama se comporta como un sultán cuando en la ficción dramática no está caracterizado como un sultán ${ }^{17}$. Sin duda alguna, aquí

\footnotetext{
13 Precisamente, para Janz, que Schiller confiera a lo trágico la equiparación con lo humano es lo que asegura la superioridad de la tragedia (1992: 1552). A nuestro juicio, Janz está convirtiendo a Schiller en un tardoromántico feurbachiano, como el Wagner de 1849.

${ }^{14}$ Como Edipo.

${ }^{15}$ Como Antígona.

16 https://ores.klassik-

stiftung.de/ords/f?p=401:2:13922666693922::NO:RP:P2_ID,P2_ANSICHT,P2_QUELLE:70152,1,70

(último acceso: 04/05/2021).

${ }^{17}$ Ciertamente el Saladino de Lessing oscila entre el despotismo (ejecutando a cruzados y tendiéndole una trampa a Nathan) y un magnánimo talante ilustrado (comprendiendo a través del relato que las tres religiones del libro constituyen una familia y una unidad). Lo que en el fondo critica Schiller es la incapacidad de Lessing para conseguir una consonancia entre lo didáctico y lo teatral.
} 
se manifiesta lo sensible que era Schiller al drama histórico, y como el motivo histórico puede ser inspirador de los dramas, pero que el personaje dramático ha de ser sometido a un procesamiento que enmarcado en el asunto y los conflictos de este ${ }^{18}$. La escritura del texto es muy resuelta. Solo hay una pequeña modificación en el borrador al originario erscheint, se le añade uns, lo que da como resultado Deßwegen [sic] erscheint uns diese Motiv plump und ganz. unpassend (Schiller, 1963: 91). Se está refiriendo al autor al motivo falsamente mixto de Saladino, y ese uns mienta al propio escritor que se erige así en instancia de crítica y juicio. En la nítida copia a limpio ese cambio se incorpora sin más incidencias.

El texto más intensamente kantiano de este repertorio es «Wohlgefallen am Schönen» (El placer por lo bello) ${ }^{19}$. Es significativo que la portadilla del texto, tanto en el borrador como en la copia en limpio ponga «Filosofía». Tanto por su temática como por el modo de tratarla, hay fuertes vínculos de contenido con la carta 26 Sobre la educación estética del ser humano. Se defiende la tesis de que el acceso del salvaje a la humanidad se produce a través de la adquisición del sentimiento de lo bello (Schiller, 1962: 399). Es decir, la sensibilidad hacia la forma pura, a la valoración del objeto por su forma y no por su posesión es lo que confirma la obtención de una humanidad. Sin duda, aquí resuenan con fuerza los ecos del primer momento del juicio sobre lo bello kantiano: el del interés desinteresado. Para fortalecer la posición del texto, se reconoce que entre los animales y los salvajes existe un sentido por el adorno y la limpieza (Schmuck und Putz). Sin embargo, este sentido no es todavía un tránsito a la humanidad pues todavía sigue siendo un proceso natural. El sujeto atento al adorno y la limpieza todavía está seducido por el brillo de los colores, se siente impulsado hacia la muestra de la vanidad o interesado en el aumento de la riqueza. Aquí Schiller está lanzando una crítica indirecta a la estética británica que confundía lo bello con lo agradable y «deducía lo bello meramente de causas físicas», tal y como el autor reprocha a Burke en sus apuntes para sus lecciones de estética (Schiller, 1963: 77). Se apunta, brevemente que el contacto con la belleza es lo que acaba creando un sentimiento hacia ella. Siendo el tránsito de esa belleza presente, pero no sentida, a esa otra que va acompañada de sentimiento un elemento indispensable para alcanzar la humanidad. Es muy rotundo Schiller al principio del texto donde señala que el gran paso que se da con la adquisición del sentimiento de lo

${ }^{18}$ El lugar donde más intentó atender Schiller a esa diferencia fue en la trilogía W allenstein, donde quiso nítidamente distinguir entre el Duque de Friedland histórico (que ya había descrito en su Historia de la guerra de los treinta años) y el de su drama.

${ }^{19}$ https://ores.klassik-stiftung.de/ords/f?p=401:2:4713872728248::::p2_ansicht:1 (último acceso: 04/05/2021). 
bello no ha quedado debidamente mostrado en ninguna historia de la humanidad (Schiller 1963: 89). En el tomo 8 de la Berliner Ausgabe, Barthold Petzold echa en cara a Schiller que, aun reclamando una atención de la historia de la humanidad a la adquisición del sentimiento de lo bello, él mismo no escribiera una detallada exposición de ese hecho. Error que se reproduce en «Methode», donde tampoco Schilller hace una historia del género humano ${ }^{20}$. (Petzold, 2005: 877). El final del escrito es una corroboración retórica de la afirmación inicial. En estas últimas líneas se exclama que fue un gran avance pasar «de las chozas con forma de cesto y las sucias tiendas de piel de animal al orden griego de columnas, a los templos y a los pórticos». Todo ello se remata señalando que ese gran avance consistió en Die Reinlichkeit (la pureza). No se puede decir que ciertos momentos de este texto se amolden a los parámetros actuales de lo políticamente correcto. La escritura, total y sorprendentemente exenta de titubeos (tachaduras, añadidos, correcciones) tanto en el boceto como en la versión para impresión, muestra a un Schiller seguro de su postura estética, si no literalmente reproductora de los planteamientos kantianos, sí notablemente fiel a ellos.

Schiller es un pensador fascinante por su capacidad de afrontar las contradicciones, no de puentearlas (o cabalgarlas). Pocos escritores de creación han estado tan dotados y han estado tan instruidos en filosofía como Schiller, pero la utilización de esas herramientas (como lleva a cabo en el texto anterior) no le impide percibir sus limitaciones. En este sentido, podría resultar contradictorio que, después de haber puesto tan de relieve en «Wohlgefallen am Schönen» la superación del estado natural, defienda los puntos de vista presentes en «Methode» ${ }^{21}$. Según este escrito, la adaptación del derecho natural, la política, la moral al sistema es directamente proporcional a la falta de aplicación de todos esos constructos teóricos al mundo a la vida y a la creación artística. ¿No será- se pregunta Schiller- porque el filósofo parte de las leyes y los principios racionales mientras que la naturaleza lo hace desde las potencias ciegas y lo fáctico? El filósofo llega a su fin «si presupone inmediatamente que el ser humano es racional» (Schiller, 1963: 90). Aquí la cláusula introducida en el borrador que modifica la redacción original es gleich («inmediatamente»). Esta introducción es muy significativa, pues supone un claro distanciamiento de una antropología racionalista. «El ser

\footnotetext{
$20 \mathrm{Si}$ bien, como veremos acto seguido, la tesis de este texto es en cierta medida contrapuesta a la de «Wohlgefallen am Schönen». Mientras que en este escrito la clave es el tránsito de la animalidad a la humanidad, en «Methode» la clave es no proponer una humanidad que excluya e ignore nuestra condición interna de animal. El primer texto se apoya en Kant y el segundo en Rousseau.

${ }^{21}$ https://ores.klassik-

stiftung.de/ords/f?p=401:2:13922666693922::NO:RP:P2_ID,P2_ANSICHT,P2_QUELLE:70151,1,70

(último acceso: 04/05/2021).
} 
humano no es racional, solo lo es tardíamente y cuando el mundo se ha configurado (o institucionalizado)». Acto seguido se apostilla que el ser humano es «poderoso, violento, él es astuto y puede ser ingenioso mucho antes de ser racional» (Schiller, 1963: 90). De nuevo dos modificaciones, er ist («él es» astuto) y kann («él puede» ser ingenioso) introducen un importante cambio en la redacción, lo que pretendía ser inicialmente una enumeración descriptiva, se convierte en una cláusula explicativa de aquello que el ser humano es y puede ser antes de ser racional. Todo ello para afirmar que la política y el derecho natural han de deducirse de la vida si es que aspiran a tener alguna influencia en la propia vida. Estos dos cambios se mantienen en la copia en limpio.

De los cinco textos comentados «Bildungsstuffen» [sic] (Niveles de formación) tiene dos características descollantes: es el más cercano a los puntos de vista definitivos de Friedrich Schiller sobre la educación estética y tiene significativas modificaciones de escritura. El escrito comienza señalando que, en sus juicios sobre el teatro y las artes plásticas, los inexpertos y los inmaduros son mucho menos comprensivos que los expertos y buenos conocedores. Y es que el que es rico y posee plenitud interna puede dar a los otros, pero el que es pobre se siente por un momento rico cuando toma algo de los otros. El carácter de escrito tentativo del texto se manifiesta en su no muy clara ordenación expositiva, pues, tras contrastar al rico entendido, y al pobre de espíritu inexperto, Schiller se refiere al que tal vez sea el nivel formativo más bajo: aquel para el que la cultura aún no ha comenzad y toda novedad, todo cambio, todo lo brillante le llama la atención (1963: 90). Parece que el orden más lógico habría sido comenzar por la descripción de este sujeto. En todo caso, está claro que, con su gran capacidad para la disección, Schiller conforme a la clasificación que lleva a cabo en las cartas «Sobre la educación estética del ser humano», está mentando, con este tercer ejemplo al individuo llevado por el impulso material (o Stofftrieb). Por su parte el «listillo» (el Klügling) es el conducido por el impulso formal (o Formtrieb) y el rico de espíritu y experto en arte y teatro es el que ha alcanzado el impulso de juego (o Spieltrieb).

Los cambios de redacción son, como ya mencionamos, muy significativos. Para empezar Schiller pretendía escribir acerca de ese ser humano de predominante impulso formal, «el que se siente (füblt) pobre», pero acaba escribiendo que «es» (ist) pobre.

Y el texto toma claramente un giro en su concepción a partir de su segundo párrafo. Parece pretenderse una exposición de las conductas que debe adoptar el artista ante el realmente entendido y el solo entendido a medias (Den Künstler wird daher...) [Imagen 2]. Sin embargo, estas palabras son tachadas, y, como ya señalamos, lo que se inicia es una 
descripción del ser humano promedio, ese al que, llevado por su sensualidad, todo lo que se le presenta, en cuanto que es nuevo, le impresiona y le agrada. En la copia a limpio no hay cambios de contenido.

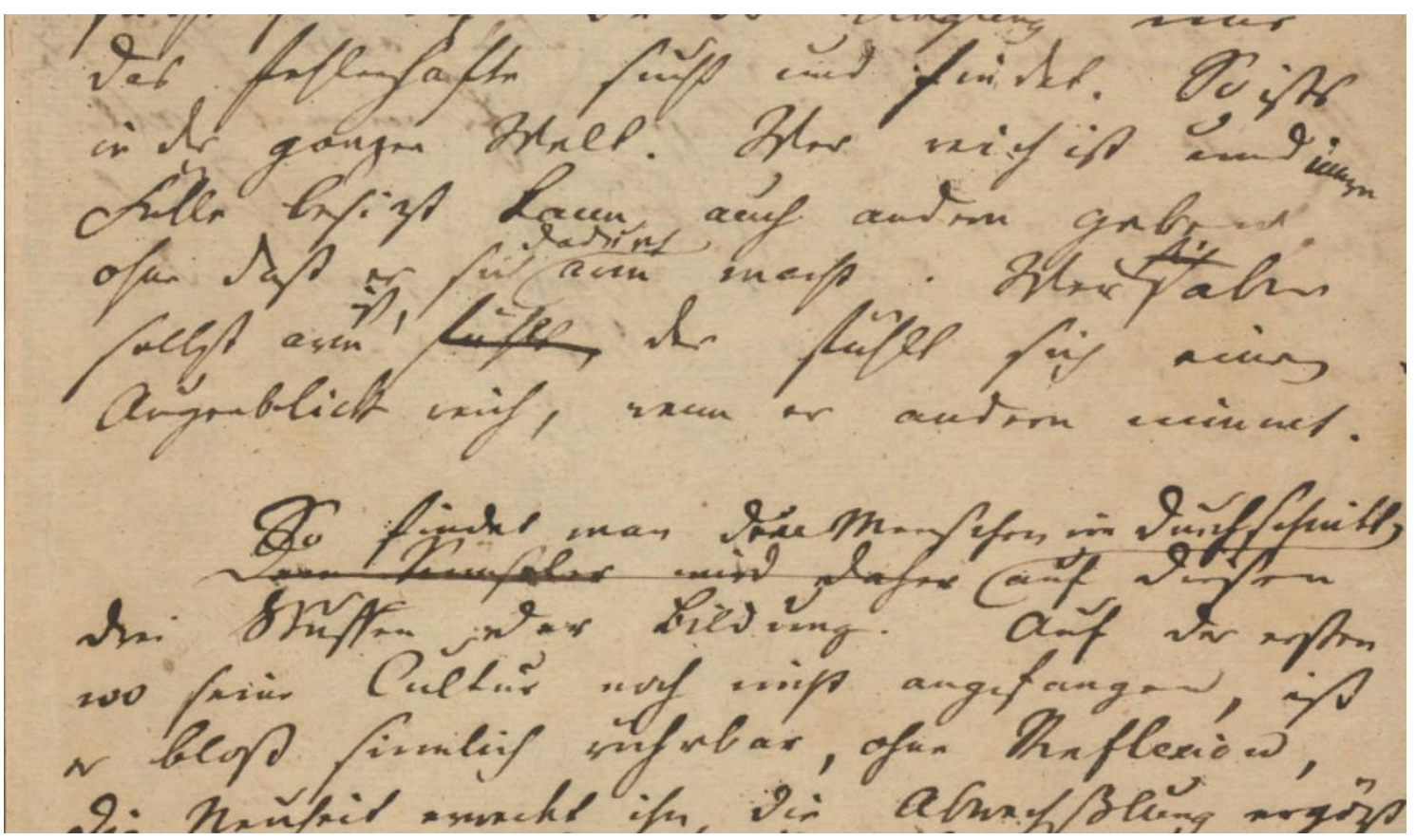

Imagen 2/ Foto: Klassik Stiftung Weimar.

Con este texto, Schiller se desmarca claramente de un clasicismo arqueológico que pretendería la vuelta a los griegos como un estadio ideal de lo humano. Ello queda confirmado en las anotaciones que hace al texto de Wilhelm von Humboldt «Über das Studium des Altertums und des Griechischen insbesondere» (Sobre el estudio de la Antigüedad, especialmente de la griega), que data, al igual que las mencionadas anotaciones, de 1793. La muy veneradora actitud de Humboldt está, o quiere estar en consonancia con la pulcra visión de la filología clásica propugnada por Friedrich August Wolf, pero recae en el idealismo del que este quería escapar. Schiller crítica precisamente la identificación abstracta de lo griego con lo perfecto. Cuando en el parágrafo 12 de su escrito Humboldt señala que es necesario «unir las aspiraciones individuales a una totalidad y concretamente a la unidad del fin más noble, es decir, de la más elevada y proporcionada formación del ser humano», Schiller repone que hay tres momentos de evolución de la humanidad: cuando el objeto está ante nosotros pero confuso y volátil, cuando somos capaces de distinguir los rasgos particulares (entonces nuestro entendimiento es claro pero estrecho de miras) y cuando conseguimos unir lo particular y la totalidad está ante nosotros, pero ya no de un modo 
confuso sino iluminado por todos los lados. «En el primer periodo estuvieron los griegos. En el segundo estamos nosotros. Se tiene esperanza en que llegue el tercero, y por eso no es deseable que retornen los griegos» (Schiller, 1992: 1075). Él opone, a una nostalgia por el retorno, una antropología progresiva.

Comparando estos textos de Schiller, de resuelta escritura en unos casos y de prurito por la corrección en otros, hemos intentado acercarnos a la evolución de su pensamiento entre 1792 y 1794. 


\section{BIBLIOGRAFÍA}

Beck, Friedrich (1991), «Die deutsche Schrift. Medium in fünf Jahrhunderten deutscher Geschichte», en Archiv für Diplomatik, 37: 453-479.

Hinderer, Walter (2009), «Die exzentrische Bahn», en Schiller und kein Ende. Metamorphosen und kreative Aneinungen, Würzburg, Königshausen \& Neumann: 59-180.

Janz, Rolf-Peter (ed.) (1992), «Kommentar», en Friedrich Schiller Theoretische Schriften, Frankfurt a. M., Deutscher Klassiker Verlag: 1125-1591.

Petzold, Barthold (2005) «Erläuterungen» en Friedrich Schiller. Sämtliche Werke. Philosophische Schriften, Berlin, Aufbau Verlag: 748-1023.

Schiller, Friedrich (1962), Werke. Nationalausgabe, Band 20, Weimar, Hermann Böhlaus Nachfolger.

Schiller, Friedrich (1963), Werke. Nationalausgabe, Band 21.2, Weimar, Hermann Böhlaus Nachfolger.

Schiller, Friedrich, (1992) «Anmerkungen zu Wilhelm von Humboldt “Über das Studium des Alterthums und des griechischen insbesondere"», en Friedrich Schiller Theoretische Schriften. Friedrich Schiller Werke und Briefe, Band 8, Frankfurt a. M., Deutscher Klassiker Verlag: 1075-1078.

Wiese, Benno von (1963) «Anmerkungen zu Notizen aus Schillers Nachlaß», en Schiller, Friedrich (1963), Werke. Nationalausgabe, Band 21.2 ..:389-396.

https://ores.klassik-stiftung.de/ords/f?p=401:2::.::P2_ID:70153 (último acceso: $01 / 05 / 2021)$

https://ores.klassikstiftung.de/ords/f?p=401:2:13922666693922::NO:RP:P2_ID,P2_ANSICHT,P2_ QUELLE:70152,1,70 (último acceso: 04/05/2021).

https://ores.klassik-stiftung.de/ords/f?p=401:2:4713872728248::::p2_ansicht:1 (último acceso: 04/05/2021).

https://ores.klassikstiftung.de/ords/f?p=401:2:13922666693922::NO:RP:P2_ID,P2_ANSICHT,P2_ QUELLE:70151,1,70 (último acceso: 04/05/2021). 
SOBRE EL AUTOR

\section{Miguel Salmerón Infante}

Es profesor titular del área de Estética en la Universidad Autónoma de Madrid, en el Departamento de Filosofía.

Contact information: correo electrónico: miguel.salmeron@uam.es 